\title{
Percepção materna do cuidado à saúde da criança na estratégia saúde da família de dois municípios da Paraíba, Brasil*
}

\author{
Mother's Perception of the Child Health Care under the Family Health Strategy in two Municipalities of \\ Paraiba, Brazil
Percepción materna del cuidado a la salud del niño en la estrategia salud de la familia de dos municipios de Paraíba, Brasil

Dixis Figueroa Pedraza ${ }^{a}$

Universidade Estadual da Paraíba, Brasil

ORCID: http://orcid.org/0000-0002-5394-828X

DOI: https://doi.org/10.11144/Javeriana.rgps17-34.pmcs

Iná da Silva Santos

Recepção: 10 Abril 2017

Universidade Federal de Pelotas, Brasil

\author{
Aprovação: 20 Outubro 2017
}

\section{Resumo:}

Dada a importância do cuidado da criança associada a sua vulnerabilidade, este estudo teve por objetivo avaliar a assistência à saúde da criança e analisar fatores associados na Estratégia Saúde da Família de dois municípios da Paraíba, Brasil. Tratou-se de uma pesquisa avaliativa dos atributos da Atenção Primária à Saúde na perspectiva materna. Para a coleta de dados utilizou-se questionário validado no Brasil. Foram atribuídos escores a quatro das dimensões da Atenção Primária à Saúde. Constatou-se que apenas a utilização apresentou valores satisfatórios em ambos os municípios. O escore da integralidade foi adequado em um dos municípios, o qual também apresentou comportamento melhor em todas as dimensões e na avaliação geral dos serviços. Crianças de maior idade e de famílias com maior renda se associaram significativamente à atribuição de baixos escores. Como principal achado encontrouse baixa qualidade de cuidados primários de saúde da criança, sobretudo na acessibilidade e longitudinalidade.

Palavras-chave: atenção primária à saúde, estratégia saúde da família, pesquisa sobre serviços de saúde, avaliação em saúde, gestão em saúde, cuidado da criança.

\section{Abstract:}

Given the importance of child care associated with its vulnerability, this study aimed to evaluate the child health care and analyze associated factors in the Family Health Strategy of two municipalities of Paraíba, Brazil. It was an evaluative research about the attributes of Primary Health Care from the perspective of mothers. Data were collected using a questionnaire validated for use in Brazil. Scores were given for four dimensions of Primary Health Care. It was found that only utilization achieved satisfactory scores in two municipalities. The integration score was adequate in one of the municipalities, which also presented better behavior in all dimensions and in the general evaluation of services. Older children and from higher income families were significantly associated with the low scores. The main finding was the low quality of primary health child care especially in accessibility and longitudinality. Keywords: primary health care, family health strategy, health services research, health evaluation, health management, child care.

\section{Resumen:}

Dada la importancia del cuidado del niño debido a su vulnerabilidad, se evaluó la asistencia a la salud del niño y factores asociados en la estrategia de salud de la familia de dos municipios de Paraíba, Brasil. Fue una investigación evaluativa de los atributos de la atención primaria de salud, según perspectiva materna, utilizando un cuestionario validado en Brasil. Se atribuyeron puntajes a cuatro de las dimensiones de la atención primaria de salud. Se constató que la utilización apenas presentó valores satisfactorios en los dos municipios. El puntaje de integralidad fue adecuado en uno de los municipios que también tuvo mejor comportamiento en todas las dimensiones y en la evaluación general de los servicios. Niños de mayor edad y de familias con mayor renta se asociaron significativamente a bajos puntajes. Como hallazgo principal se encontró baja calidad de cuidados primarios de salud al niño, sobre todo en accesibilidad y longitudinalidad.

Palabras clave: atención primaria de salud, estrategia salud de la familia, investigación en servicios de salud, evaluación en salud, gestión en salud, cuidado del niño.

\footnotetext{
a Autor de correspondência. Correio eletrônico: dixisfigueroa@gmail.com
} 


\section{Introdução}

O surgimento da Atenção Primária à Saúde (APS) relaciona-se à necessidade de mudança organizacional dos serviços de saúde com foco na cura e reabilitação para a promoção da saúde e prevenção de doenças [1]. Nessa concepção, a APS institui-se por atributos essenciais (atenção ao primeiro contato, longitudinalidade, integralidade e coordenação) e derivados (atenção centrada na família, orientação comunitária e competência cultural) [2,3]. Essa organização relaciona-se a melhores condições de cuidado, fluxo dos usuários dentro do sistema, utilização de práticas preventivas, satisfação dos usuários, indicadores de saúde e iniquidades [3].

Dentre os instrumentos disponíveis para avaliar a APS, o Primary Care Assessment Tool (PCATool) destaca-se por sua capacidade de medir a presença e extensão dos atributos da assistência, por possuir componentes específicos para fases do ciclo da vida e por incluir aspectos de estrutura, processo e resultados que são determinantes da qualidade dos cuidados [1,2]. Considera-se que avaliações por meio do PCATool possibilitem comparar e sistematizar resultados sobre o desempenho dos serviços de saúde em contextos diferentes $[1,4,5]$.

No Brasil, a Estratégia Saúde da Família (ESF) constitui a principal alternativa de reorganização da APS $[3,4,5]$. As avaliaçóes da Estratégia, apesar do uso de instrumentos diferentes [5], sugerem fragilidades que questionam seu caráter substitutivo, a exemplo de precariedade no desenvolvimento tecnológico, gerencial e científico; visão vertical dos programas; omissão de política trabalhista adequada; uso rotineiro exagerado dos recursos; e não apropriação das equipes de saúde em termos de completude, funcionalidade e formação dos profissionais $[5,6]$. Resultados adicionais são necessários para a compreensão mais apurada dessa conjuntura e dos desafios impostos, sobretudo por meio do PCATool que predomina como instrumento de avaliação $[2,4,5]$. Para a população infantil, a avaliação da APS pode ser especialmente relevante à compreensão das internações por condições sensíveis à atenção primária como consequência da eficiência do serviço [7].

Este estudo objetivou avaliar a assistência à saúde da criança e analisar fatores associados na ESF de dois municípios do estado da Paraíba.

\section{Métodos}

\section{Tipo de estudo}

Trata-se de uma pesquisa de cunho transversal para avaliar os atributos da APS relacionados aos cuidados de crianças menores de cinco anos atendidas por equipes de saúde da família no Brasil.

\section{Universo, população e amostra}

O estudo foi conduzido em dois munícipios da Paraíba, Nordeste do Brasil. A escolha dos municípios considerou suas semelhanças quanto a posição geográfica (localização na região metropolitana da capital do Estado com acesso à rede de serviços nela disponibilizados), grau de urbanização (de quase 100\%), indicadores demográfico-sociais (índice de desenvolvimento humano de nível médio - 0.748 no Município 1 e 0.649 no Município 2) e tradição na organização de serviços primários de saúde (cobertura populacional da ESF de quase 100\%). O Município 1 tem população de 57944 habitantes, dos quais 4596 são crianças menores de cinco anos, e possui sistema de saúde composto por 19 equipes da ESF. O Município 2 tem população de 99716 habitantes, dos quais 7862 são crianças menores de cinco anos, e possui sistema de saúde composto por 28 equipes da ESF. Entretanto, no Município 1 o número de profissionais de saúde (médicos, pediatras, 
enfermeiros, nutricionistas), para cada 1000 habitantes, no Sistema Único de Saúde, é maior do que no Município 2.

A população de estudo foi composta por mães de crianças menores de cinco anos atendidas por equipes da ESF dos municípios em estudo. Para o cálculo do tamanho amostral foram empregados parâmetros que resultaram da avaliação da qualidade da atenção à saúde da criança na ESF, conforme a visão de usuários no município de Montes Claros, Minas Gerais [8]. No estudo, constatou-se avaliação negativa das equipes formadas por médicos e enfermeiros com residência em Saúde da Família de 41.9\%, e de 58.1\% daquelas compostas por profissionais sem esta formação específica. Assim, considerando-se prevalência esperada de avaliação negativa dos serviços de saúde pelos usuários de 41.9\%, no Município 1 (com melhor composição de profissionais de saúde), e de $58.1 \%$, no Município 2, nível de $95 \%$ de confiança, poder de $80 \%, 10 \%$ de acréscimo para compensar eventuais perdas e efeito do desenho de 1.2, obteve-se a necessidade de entrevistar 157 sujeitos por município.

A amostra foi selecionada por conglomerados em dois estágios. No primeiro, foram sorteadas aleatoriamente as equipes de saúde da família e no segundo estágio um número fixo de mães de forma intencional. Em cada município foram selecionadas nove equipes da ESF, que representaram cerca da metade das equipes do Município 1 e 1/3 das do Município 2. Cada equipe contribuiu, em média, com 18 mães para o total da amostra que obedeceu a dois critérios: i. uma amostra intencional, composta por todos os atendimentos nas consultas de puericultura no dia da coleta de dados, em um dia típico de trabalho; ii. uma amostra de crianças encaminhadas aos serviços de saúde pelos Agentes Comunitários de Saúde, conforme rotina de trabalho, das quais selecionaram-se aletoriamente a quantidade que permitisse completar o total previsto para cada equipe de saúde. No final do período de coleta de dados, a amostra constituiu-se de 321 mães de crianças menores de cinco anos, das quais 153 do Município 1 e 168 do Município 2.

\section{Fontes e instrumentos de coleta de dados}

Para a coleta de dados foram aplicados dois instrumentos às mães das crianças: um com dados sociodemográficos das famílias e outro validado (PCATool) [9] para apreender a percepção materna dos serviços de saúde prestados pelas equipes da ESF aos seus filhos menores de cinco anos. O questionário da situação sociodemográfica contemplou as seguintes informações: sexo e idade da criança, idade e alfabetização materna, condições do domicílio em relação à presença de esgoto domiciliar e número de pessoas, benefício do Programa Bolsa Família e renda familiar. Além disso, a segurança alimentar da família foi medida por meio da Escala Brasileira de Insegurança Alimentar [10].

$\mathrm{Na}$ validação do PCATool para sua aplicação em crianças, foram identificadas oito dimensões com o $\alpha$ de Cronbach variando de 0.74 a 0.88 por análise fatorial. Assim, o instrumento ficou constituído por 45 itens e oito atributos (acesso de primeiro contato, continuidade, coordenação, três atributos de integralidade, orientação familiar e comunitária). Estes resultados indicaram adequada validade e confiabilidade do PCATool para seu uso na avaliação dos serviços da APS para crianças [11].

O PCATool [9] foi usado na versão destinada às crianças, contemplando os componentes de acesso de primeiro contato - utilização, acesso de primeiro contato - acessibilidade, longitudinalidade e integralidade serviços prestados. As respostas do instrumento são do tipo Likert [12], com escore de 1 a 4 para cada elemento que compõe o atributo: com certeza sim [4]; provavelmente sim [3]; provavelmente não [2]; com certeza não [1]. Para as perguntas C2, C4, C5 e D10, por serem elaboradas de maneira inversa (quanto maior o valor, menor a orientação para a atenção primária), os seus valores foram invertidos (valor $4=1$, valor $3=2$, valor $2=3$, valor $1=4$ ), conforme especificações do instrumento. Para cada atributo, foram calculados os escores e a partir dos mesmos o escore geral. Nos casos de respostas para apenas três atributos, o cálculo do escore geral desconsiderou o atributo não avaliado. Mães que responderam apenas as questões de dois dos atributos em análise foram excluídas, o que correspondeu a três casos no Município 1 e 11 no Município 2 e totalizou 307 
questionários válidos para as análises. Após consolidação das respostas para cada atributo, os valores foram transformados em uma escala contínua, variando entre zero e dez, por meio da fórmula: Escore ajustado $=$ [(escore obtido-1) / [4-1]] x 10. Valores de escore geral $<6.6$ foram considerados baixos ou insatisfatórios (menores do que três segundo a escala Likert) [12].

A coleta foi realizada nas unidades de saúde entre julho e dezembro de 2014. A equipe foi formada por profissionais e estudantes da área de saúde, com experiência prévia em trabalho de campo, o qual foi supervisionado por profissional capacitado. $\mathrm{O}$ controle de qualidade do estudo incluiu: treinamento e padronização dos entrevistadores, construção de Manual de Instruções e teste prévio dos entrevistadores e do questionário socioeconômico.

\section{Processamento e análise dos dados}

Os dados coletados foram organizados em planilhas eletrônicas e digitados em dupla entrada. O aplicativo Validate do software Epi Info versão 3.3.2 foi usado para analisar a consistência dos dados gerando o banco final que foi utilizado nas análises estatísticas.

Nas comparações entre os municípios, utilizaram-se os testes qui-quadrado e " $t$ " de Student para analisar diferenças nas proporções das características sociodemográficas e nas médias dos escores de avaliação dos atributos, respectivamente. A associação das variáveis independentes (características sociodemográficas) com a variável dependente (escore geral da avaliação materna dos serviços de saúde) foi verificada por meio do teste do qui-quadrado, enquanto diferenças entre municípios nas proporções de baixo escore geral segundo categorias das variáveis independentes foram analisadas por meio do teste de igualdade de proporçóes (teste com estatística Z).

A Regressão de Poisson com variância robusta utilizou-se para estimar as razões de prevalência (RP) e seus respectivos intervalos de confiança de 95\% (IC95\%) para avaliar a associação entre as características sociodemográficas e o escore geral insatisfatório dos serviços de saúde. $\mathrm{O}$ modelo explicativo considerou as variáveis com nível de significância de até $25 \%(\mathrm{p}<0.25)$ nas análises bivariadas. A presença de confusão e interação foi explorada a partir de modelos de regressão, realizada com procedimento backward. Partiu-se de um modelo saturado contendo todas as potenciais variáveis modificadoras de efeito e seus respectivos produtos-termo, além das candidatas a confundimento. Consideraram-se modificadoras de efeito as variáveis que apresentaram resultados estatisticamente significantes $(\mathrm{p}<0.05)$, segundo o teste da razão de máxima verossimilhança. As variáveis de confusão foram aquelas que produziram alteração abaixo da média na medida pontual das outras associações testadas.

Em todas as análises, o nível de significância admitido foi de 5\%. Utilizou-se o software Stata versão 12.0.

\section{Aspectos éticos}

A pesquisa foi aprovada pelo Comitê de Ética em Pesquisa da Universidade Estadual da Paraíba, com o protocolo de número 19689613.3.0000.5187. Todos os participantes da pesquisa assinaram o Termo de Consentimento Livre e Esclarecido como condição necessária e concordância relacionada à participação no estudo.

\section{Resultados}

O perfil sociodemográfico das famílias envolvidas no estudo está compendiado na Tabela 1. Em ambos os municípios, a maioria das crianças possuía idade inferior a 25 meses. Observaram-se frequências maiores do 
que 50.0\% para ausência de rede pública de esgoto domiciliar, mínimo de quatro pessoas no domicílio, renda familiar mensal inferior a dois salários mínimos e benefício do Programa Bolsa Família. As frequências de insegurança alimentar moderada/grave foram de 41.3\%, no Município 1, e 49.7\%, no Município 2. Nenhuma das variáveis relacionadas à situação sociodemográfica apresentou diferença entre os municípios. Nessa mesma tabela, observa-se que a proporção de mães que avaliaram positivamente a APS foi superior no Município 1 (54.0\%) quando comparado com o Município 2 (24.2\%) (Tabela 1). 
TABELA 1

Características sociodemográficas e avaliação materna da Atenção Primária à Saúde no contexto da Estratégia Saúde da Família em dois municípios do Estado da Paraíba, 2014

\begin{tabular}{|c|c|c|c|c|c|}
\hline \multirow[t]{2}{*}{ Variáveis } & \multicolumn{2}{|c|}{ Município 1} & \multicolumn{2}{|c|}{ Municipio 2} & \multirow[t]{2}{*}{$\mathbf{p}^{*}$} \\
\hline & $\mathbf{n}$ & $\%$ & $\mathbf{n}$ & $\%$ & \\
\hline \multicolumn{5}{|l|}{ Sexo da criança } & 0.330 \\
\hline Masculino & 80 & 53.3 & 75 & 47.8 & \\
\hline Feminino & 70 & 46.7 & 82 & 52.2 & \\
\hline \multicolumn{5}{|l|}{ Idade da criança (meses) } & 0.063 \\
\hline $25-60$ & 30 & 20 & 48 & 30.6 & \\
\hline$<25$ & 120 & 80 & 109 & 69.4 & \\
\hline \multicolumn{5}{|l|}{ Idade materna (anos) } & 0,295 \\
\hline$\geq 21$ & 117 & 78.5 & 113 & 73.4 & \\
\hline$<21$ & 32 & 21.5 & 41 & 26.6 & \\
\hline \multicolumn{5}{|l|}{ Alfabetização materna } & 0.825 \\
\hline $\begin{array}{l}\text { Sabe ler, escrever e fazer contas por } \\
\text { escrito }\end{array}$ & 120 & 80 & 124 & 79 & \\
\hline $\begin{array}{l}\text { Nào sabe ler, escrever nem fazer } \\
\text { contas por escrito }\end{array}$ & 30 & 20 & 33 & 21 & \\
\hline \multicolumn{5}{|l|}{ Trabalho materno fora de casa } & 0.211 \\
\hline $\operatorname{Sim}$ & 31 & 20.7 & 42 & 26.8 & \\
\hline Não & 119 & 79.3 & 115 & 73.2 & \\
\hline \multicolumn{5}{|l|}{ Esgoto domiciliar } & 0.514 \\
\hline Rede pública & 28 & 18.7 & 34 & 21.7 & \\
\hline Outros & 122 & 81.3 & 123 & 78.3 & \\
\hline \multicolumn{5}{|l|}{ Número de pessoas no domicilio } & 0.781 \\
\hline$<4$ & 50 & 33.3 & 50 & 31.8 & \\
\hline$\geq 4$ & 100 & 66.7 & 107 & 68.2 & \\
\hline \multicolumn{5}{|l|}{ Beneficio do Programa Bolsa Familia } & 0.085 \\
\hline $\operatorname{Sim}$ & 84 & 56 & 103 & 65.6 & \\
\hline Não & 66 & 44 & 54 & 34.4 & \\
\hline \multicolumn{5}{|l|}{ Renda Familiar } & 0.806 \\
\hline$>=2 \mathrm{SM}$ & 41 & 27.7 & 41 & 26.5 & \\
\hline$<2 \mathrm{SM}$ & 107 & 72.3 & 114 & 73.5 & \\
\hline \multicolumn{5}{|l|}{ Segurança Alimentar Familiar } & 0.142 \\
\hline Segurança alimentar/insegurança & 88 & 58.7 & 79 & 50.3 & \\
\hline
\end{tabular}

SM: Salário Mínimo (R \$24,00); * Teste do qui-quadrado para proporções; ${ }^{* *}$ Escore Geral da Atenção Primária à Saúde. 
Os escores dos atributos da APS para os dois municípios estão na Tabela 2. Ambos os municípios apresentaram valores de escores satisfatórios $(\geq 6.6)$ para a utilização, e insatisfatórios $(<6.6)$ para a acessibilidade e a longitudinalidade. $\mathrm{O}$ escore da integralidade (ações de promoção e prevenção) foi satisfatório no Município 1 e insatisfatório no Município 2. Para todos os atributos, as médias dos escores foram superiores no Município 1. Segundo critérios específicos, apenas o encaminhamento a especialista, na utilização, e o tempo de espera para consultas, na acessibilidade, não apresentaram diferenças entre os municípios. $\mathrm{Na}$ análise conjunta dos atributos, os valores de escore geral foram $<6.6$ nos dois municípios, sendo significativamente mais elevado no Município 1 (Tabela 2). 
TABELA 2

Escores médios dos atributos da Atenção Primária à Saúde, segundo percepção materna de crianças menores de cinco anos de idade no contexto da Estratégia Saúde da Família em dois municípios do Estado da Paraíba, 2014

\begin{tabular}{|c|c|c|c|}
\hline \multirow[t]{2}{*}{ Atributos } & $\begin{array}{c}\text { Municipio } 1 \\
(n=157)\end{array}$ & $\begin{array}{c}\text { Municipio } 2 \\
(\mathrm{n}=150)\end{array}$ & \multirow[t]{2}{*}{$p^{*}$} \\
\hline & Média:dp & Média $\pm d p$ & \\
\hline Utilização & $7.96 \pm 0.206$ & $6.61 \pm 0.232$ & $<0.001$ \\
\hline Por consulta de revisão & $3.58 \pm 0.068$ & $3.03 \pm 0.096$ & $<0.001$ \\
\hline Por novo problema de saúde & $3.21 \pm 0.091$ & $2.66 \pm 0.103$ & $<0.001$ \\
\hline Encaminhamento a especialista & $3.37 \pm 0.082$ & $3.23 \pm 0.084$ & 0.228 \\
\hline Acessibilidade & $5.02 \pm 0.190$ & $4.13 \pm 0.187$ & 0.001 \\
\hline Atendimento no mesmo dia & $2.89 \pm 0.094$ & $2.59 \pm 0.095$ & 0.024 \\
\hline Tempo de marcação de consulta & $2.59 \pm 0.099$ & $2.39 \pm 0.099$ & 0.149 \\
\hline Marcação para consulta de revisão & $3.09 \pm 0.089$ & $2.41 \pm 0.096$ & $<0.001$ \\
\hline Tempo de espera para a consulta & $1.73 \pm 0.090$ & $1.84 \pm 0.090$ & 0.402 \\
\hline Atendimento por necessidade & $2.78 \pm 0.099$ & $2.28 \pm 0.099$ & $<0.001$ \\
\hline Aconselhamento por telefone & $1.95 \pm 0.076$ & $1.93 \pm 0.081$ & 0.877 \\
\hline Longitudinalidade & $6.49 \pm 0.148$ & $4.92 \pm 0.157$ & $<0.001$ \\
\hline Rotatividade & $3.50 \pm 0.077$ & $2.97 \pm 0.092$ & $<0.001$ \\
\hline Possibilidade de comunicação telefônica & $1.95 \pm 0.086$ & $1.60 \pm 0.072$ & 0.002 \\
\hline Compreensão do usuário pelos profissionais & $3.70 \pm 0.053$ & $3.44 \pm 0.071$ & 0.004 \\
\hline Compreensão dos profissionais pelo usuário & $3.77 \pm 0.050$ & $3.09 \pm 0.069$ & 0.023 \\
\hline Disponibilidade de tempo para conversar & $3.65 \pm 0.066$ & $3.09 \pm 0.099$ & $<0.001$ \\
\hline Confiança & $3.73 \pm 0.060$ & $3.28 \pm 0.080$ & $<0.001$ \\
\hline Conhecimento da criança como pessoa & $2.76 \pm 0.100$ & $2.47 \pm 0.102$ & 0.042 \\
\hline Conhecimento da criança como paciente & $3.17 \pm 0.088$ & $2.40 \pm 0.107$ & $<0.001$ \\
\hline Conhecimento dos medicamentos da criança & $3.08 \pm 0.094$ & $2.38 \pm 0.106$ & $<0.001$ \\
\hline Vontade de mudar para outro serviço & $3.03 \pm 0.100$ & $2.29 \pm 0.107$ & $<0.001$ \\
\hline Conhecimento da familia & $2.29 \pm 0.106$ & $1.88 \pm 0.094$ & 0.003 \\
\hline Conhecimento dos problemas da familia & $2.51 \pm 0.102$ & $2.03 \pm 0.098$ & 0.001 \\
\hline Conhecimento sobre o trabalho da familia & $1.79 \pm 0.090$ & $1.38 \pm 0.063$ & $<0.001$ \\
\hline $\begin{array}{l}\text { Conhecimento sobre dificuldades para pagar } \\
\text { medicamentos que a criança precisa }\end{array}$ & $2.35 \pm 0.104$ & $1.87 \pm 0.090$ & 0.001 \\
\hline $\begin{array}{l}\text { Integralidade - serviços prestados } \\
\text { (orientações) }\end{array}$ & $6.80 \pm 0.266$ & $4.79 \pm 0.289$ & $<0.001$ \\
\hline Sobre segurança no lar & $3.57 \pm 0.078$ & $2.89 \pm 0.110$ & $<0.001$ \\
\hline
\end{tabular}


A prevalência de baixo escore geral foi maior entre as famílias com crianças de 25-60 meses de idade (76.9\%) em comparação aquelas com idade inferior aos 25 meses (55.9\%); bem como entre as famílias com renda familiar $>=2$ salários mínimos (73.2\%) quando comparadas aquelas com renda familiar $<2$ salários mínimos (56.6\%). Essas mesmas diferenças foram constatadas para a idade da criança no Município 2 e para a renda familiar no Município 1 . Segundo os resultados da estatística Z, a condição materna de não saber ler, escrever nem fazer contas; a ausência de rede pública de esgoto no domicílio e a renda familiar < 2 salários mínimos foram as categorias que atuaram isoladamente como condições discriminantes das diferenças entre os municípios nas proporções de escore geral baixo (Tabela 3). 
TABELA 3

Prevalências (\%) de baixo escore geral da Atenção Primária à Saúde segundo características sociodemográficas no contexto da Estratégia Saúde da Família em dois municípios do Estado da Paraíba, 2014

\begin{tabular}{|c|c|c|c|c|c|c|c|}
\hline \multirow[b]{2}{*}{ Variáveis } & \multicolumn{2}{|c|}{ Total } & \multicolumn{2}{|c|}{ Município 1} & \multicolumn{2}{|c|}{ Município 2} & \multirow[b]{2}{*}{$\mathrm{p}^{* *}$} \\
\hline & $\%$ & $p^{*}$ & $\%$ & $\mathbf{p}^{*}$ & $\%$ & $p^{*}$ & \\
\hline Sexo da criança & & 0.234 & & 0.470 & & 0.121 & \\
\hline Masculino & 64.5 & & 48,8 & & 81.3 & & $<0.001$ \\
\hline Feminino & 57.9 & & 42.9 & & 70.7 & & 0.006 \\
\hline Idade da criança (meses) & & 0.001 & & 0.190 & & 0.007 & \\
\hline $25-60$ & 76.9 & & 56.7 & & 89.6 & & $<0.001$ \\
\hline$<25$ & 55.9 & & 43.3 & & 69.7 & & 0.022 \\
\hline Idade materna (anos) & & 0.694 & & 0.576 & & 0.624 & \\
\hline$\geq 21$ & 60.4 & & 44.4 & & 77.0 & & $<0.001$ \\
\hline$<21$ & 63 & & 50 & & 73.2 & & 0.042 \\
\hline Alfabetização materna & & 0.321 & & 0.190 & & 0.995 & \\
\hline $\begin{array}{l}\text { Sabe ler, escrever e fazer contas por } \\
\text { escrito }\end{array}$ & 66.7 & & 56.7 & & 75.8 & & 0.061 \\
\hline $\begin{array}{l}\text { Não sabe ler, escrever nem fazer contas } \\
\text { por escrito }\end{array}$ & 59.8 & & 43.3 & & 75.8 & & $<0.002$ \\
\hline Trabalho materno fora de casa & & 0.846 & & 0.610 & & 0.725 & \\
\hline Sim & 60.3 & & 41.9 & & 73.8 & & 0.003 \\
\hline Nà̀o & 61 & & 47.1 & & 76.5 & & 0.005 \\
\hline Esgoto domiciliar & & 0.376 & & 0.083 & & 0.423 & \\
\hline Rede pública & 66.1 & & 60.7 & & 70.6 & & 0.514 \\
\hline Outros & 60 & & 42.6 & & 77.2 & & $<0.001$ \\
\hline Número de pessoas no domicilio & & 0.150 & & 0.164 & & 0.401 & \\
\hline$<4$ & 67 & & 54 & & 80 & & 0.033 \\
\hline$\geq 4$ & 58.5 & & 42 & & 73.8 & & 0.003 \\
\hline Beneficio do Programa Bolsa Familia & & 0.716 & & 0.063 & & 0.449 & \\
\hline Sim & 60.4 & & 39.3 & & 77.7 & & $<0.001$ \\
\hline Não & 62.5 & & 54.5 & & 72.2 & & 0.048 \\
\hline Renda Familiar & & 0.008 & & 0.001 & & 0.656 & \\
\hline$>=2 \mathrm{SM}$ & 73.2 & & 68.3 & & 78 & & 0.806 \\
\hline$<2 \mathrm{SM}$ & 56.6 & & 37.4 & & 74.6 & & $<0.001$ \\
\hline Segurança Alimentar Familiar & & 0.140 & & 0.873 & & 0.148 & \\
\hline Segurança alimentar/insegurança & 57.5 & & 45.5 & & 70.9 & & 0.043 \\
\hline
\end{tabular}

SM: Salário Mínimo (R \$724,00); * Teste do qui-quadrado para proporções; ${ }^{* *}$ Teste de igualdade de proporções (teste com estatística Z). 
As análises da Regressão de Poisson apresentadas na Tabela 4 confirmaram os resultados não ajustados. Mães de crianças de 25-60 meses de idade atribuíram piores escores aos atributos da APS do que as mães das crianças menores de 25 meses $(\mathrm{RP}=1.42$ e IC95\%=1.21-1.93). Por sua vez, o baixo escore geral da APS foi mais frequente entre crianças de famílias com renda $>=2$ salários mínimos do que entre crianças de famílias com renda familiar $<2$ salários mínimos $(\mathrm{RP}=1.30$ e IC95\%=1.11-2.39) (Tabela 4).

TABELA 4

Modelo ajustado do baixo escore geral da Atenção Primária à Saúde segundo características sociodemográficas no contexto da Estratégia

Saúde da Família em dois municípios do Estado da Paraíba, 2014

\begin{tabular}{|l|c|c|}
\hline \multirow{2}{*}{ Variáveis } & \multicolumn{2}{|c|}{ Baixo escore geral da Atenção Primária à Saúde $(<\mathbf{6 , 6 )}$} \\
\cline { 2 - 3 } & RP ajustada & IC95 \% \\
\hline Idade da criança (meses) & & $1.21-1.93$ \\
\hline $25-60$ & 1.42 & - \\
\hline$<25$ & 1 & $1.11-2.39$ \\
\hline Renda Familiar & & - \\
\hline$>=2 \mathrm{SM}$ & 1.30 & \\
\hline$<2 \mathrm{SM}$ & 1 & \\
\hline
\end{tabular}

SM: Salário Mínimo (R $\$ 724,00)$.

\section{Discussão}

O presente estudo permitiu aferir a percepção de mães sobre a qualidade dos serviços primários de saúde oferecidos aos seus filhos menores de cinco anos, por meio de instrumento validado. Os achados ressaltam, primeiramente, que trata-se da avaliação de famílias socioeconomicamente vulneráveis, o que corresponde com o perfil da população que preferencialmente utiliza a ESF $[12,13]$. A proximidade da moradia às unidades de saúde pode estar relacionada à escolha dos serviços oferecidos na ESF para acompanhar a saúde da criança [12].

Resultados de pesquisas anteriores têm destacado avaliações abaixo do esperado para a atenção à saúde da criança na ESF $[12,14,15,16,17]$, o que foi similarmente constatado nos dois municípios do atual estudo. A compreensão desses achados deve ser reforçada segundo indícios sobre a redução das iniquidades em saúde e melhorias do perfil de morbidade, inclusive a hospitalização por condições sensíveis $[3,18]$.

Em relação aos escores de cada atributo da APS, a utilização destacou-se por alcançar valores altos nos dois municípios, enquanto, com exceção da integralidade no Município 1, todos os outros atributos foram marcados pelos baixos escores. Esses resultados convergem com os de estudos anteriores específicos para os cuidados direcionados às crianças $[14,16,17,19]$, bem como com a avaliação geral da ESF que tem destacado avanços importantes no componente demanda, não observados no enfoque às necessidades de saúde [5].

No atributo de acessibilidade, os baixos escores observados indicam que ainda existem barreiras que dificultam o acesso das crianças aos serviços de saúde. Esse fato também foi identificado em estudos semelhantes $[12,14,16,17,19,20]$. O tempo de espera para a consulta foi o critério com pior avaliação, o 
que vem sendo apontado como um dos principais motivos de insatisfação dos usuários do SUS [21]. Além de promover uma percepção negativa sobre o atendimento, longos períodos de espera podem, particularmente, prejudicar a saúde da criança, uma vez que nessa fase da vida adoecer é mais frequente, principalmente por afecções agudas [12].

Embora com escore superior à acessibilidade, a presença da longitudinalidade também mostrou-se insatisfatória, concordando com resultados de pesquisas similares [14, 17]. No entanto, há relatos de estudos nos quais a longitudinalidade tem sido avaliada positivamente [12, 15, 22, 23]. A garantia da longitudinalidade na infância constitui um fator essencial como fonte regular de atenção à saúde associado a maior vínculo e responsabilização $[3,18]$. Nesse sentido, cabe ressaltar a importância da formação dos profissionais de saúde ser adequada às condições de vida e saúde da população para o alcance desse atributo [3]. No Brasil, a qualificação e capacitação dos profissionais de saúde constitui, consensualmente, um dos aspectos críticos do processo de transformação do modelo assistencial [1,24], caracterizando, assim, prejuízos na continuidade do cuidado. Segundo a percepção das mães deste estudo, os aspectos da longitudinalidade relacionados ao conhecimento dos profissionais sobre as características das famílias estiveram entre os de menor escore, fato que enfatiza o desafio que esse problema impõe e destaca possíveis consequências negativas no atributo de orientação familiar.

Adicionalmente, há que destacar que o comprometimento da longitudinalidade prejudica a produção de diagnósticos e tratamentos precisos, levando a encaminhamentos desnecessários para especialistas ou para a realização de procedimentos de maior complexidade $[3,18]$. Nessa conjuntura, os altos índices de Internações por Condições Sensíveis à Atenção Primária entre as crianças brasileiras menores de cinco anos questionam a efetividade da APS, associado a problemas na continuidade da atenção [25, 26]. A necessidade de maior interação com a família e com a história de vida da criança reverte à longitudinalidade mais importância, no que se refere à assistência à saúde da criança $[12,18]$.

Em relação à dimensão da integralidade - serviços prestados, observou-se valor do escore aquém do ideal no Município 2, o que também foi observado em estudos desenvolvidos em outras localidades do país [15, 17, $20,22,27]$. Sugere-se que a integralidade da assistência seja um dos atributos mais comprometidos do SUS [5], sendo questionada com base nos modos antagônicos de pensar e agir que feormam parte das práticas dos profissionais de saúde no contexto da APS [28,29]. A integralidade implica na necessidade de potencializar formas mais amplas de intervir em saúde com capacidade de ações intersetoriais e de atuação sobre seus determinantes sociais [3]. No cuidado à criança na APS, características pessoais e do processo de trabalho dos profissionais de saúde são reconhecidos como fatores importantes das práticas integrais de cuidado [18]. Esses argumentos permitem recomendar a necessidade de ações de orientação às mães relacionadas à criança, principalmente sobre crescimento, desenvolvimento e saúde que foram as que atingiram menores escores no Município 2.

A pesar da importância de compreender os fatores que determinam diferenças no desempenho da APS em contextos geográficos diferentes [24], percebe-se uma literatura carente para esses fins. Os resultados deste estudo apontaram piores escores da ESF entre as famílias com melhores rendimentos, o que pode ser justificado pela maior dependência dos serviços entre famílias mais vulneráveis, como sugerido por outros pesquisadores que obtiveram resultados similares analisando a situação socioeconômica da família [15]. Explicação similar pode ser presumida em relação aos melhores escores atribuídos pelas mães de crianças de menor idade, uma vez que nesse grupo se prescinde de maior quantidade de consultas, seja de rotina ou por maior acometimento de adoecimentos.

Outros dois estudados também analisaram a associação das condições socioeconômicas com a avaliação da APS, segundo a percepção de cuidadores das crianças. Neles, a escolaridade materna mostrou-se associada positivamente com a avaliação dos serviços $[8,12]$, o que não foi constatado no atual trabalho. No entanto, os achados deste estudo indicaram diferenças nas proporções de baixo escore geral da ESF explicadas pelos riscos condicionados à pior situação de escolaridade materna, bem como a menores rendimentos e saneamento 
domiciliar inadequado. Revela-se, assim, a importância de discriminar a qualidade dos serviços considerando essas condições e implica na necessidade de ações focalizadas que direcionem esforços relacionados à minimização de tais efeitos.

Considerando que a qualidade do cuidado é resultante de uma complexa interação de fatores, os resultados deste estudo devem ser interpretados ponderando a limitação imposta pela assunção apenas da faceta relacionada aos usuários. Apesar da importância da avaliação dos usuários na medida em que constitui o grupo que efetivamente experimenta o serviço, em seus aspectos positivos e negativos, e que tende a realizar uma avaliação mais crítica, os profissionais possuem conhecimentos científicos que podem revelar e valorizar características inerentes à dinâmica social que expressem necessidades potenciais efetivas à qualidade.

\section{Conclusões}

Os resultados apresentados apontam a necessidade de melhorar a qualidade da APS em termos de acessibilidade, longitudinalidade e integralidade. Essa melhoria implica em reformulações de alguns aspectos na organização dos serviços, no vínculo dos profissionais com as famílias e no processo de trabalho relacionado com a oferta de ações de promoção e prevenção.

\section{Contribuições}

DFP contribuiu na concepção, análise e interpretação dos dados; redação do artigo e revisão crítica do conteúdo intelectual; aprovação final da versão a ser publicada. ISS contribuiu na interpretação dos dados; revisão crítica do conteúdo intelectual; aprovação final da versão a ser publicada.

\section{Agradecimentos}

Ao Conselho Nacional de Desenvolvimento Científico e Tecnológico e à Fundação de Apoio à Pesquisa do Estado da Paraíba, pelo financiamento da pesquisa. Ao Conselho Nacional de Desenvolvimento Científico e Tecnológico e à Comissão de Aperfeiçoamento de Pessoal do Nível Superior, pela concessão de bolsas. Título do projeto: Avaliação das ações de alimentação e nutrição no marco da Estratégia Saúde da Família em municípios do estado da Paraíba. Data de inicio: 11-2013. Data de finalização: 10-2016. Financiamento: Conselho Nacional de Desenvolvimento Científico e Tecnológico (476520/2013-4. Termo assinado em 08/11/2013) e Fundação de Apoio à Pesquisa do Estado da Paraíba (37/13. Termo assinado em 01/03/2014).

Instituição de Execução: Universidade Estadual da Paraíba. Instituições envolvidas: Secretaria Municipal de Saúde de Bayeux (PB), Secretaria Municipal de Saúde de Cabedelo (PB). Conflito de interesses: Não há.

\section{Referências}

1. Savassi LCM. Qualidade em serviços públicos: os desafios da atenção primária. Rev Bras Med Fam Comunidade. 2012; 7(23):69-74.

2. Fracolli LA, Gomes MFP, Nabão FRZ, Santos MS, Cappellini VK, Almeida ACC. Instrumentos de avaliação da Atenção Primária à Saúde: revisão de literatura e metassíntese. Cienc Saude Colet. 2014; 19(12):4851-60.

3. Oliveira MAC, Pereira IC. Atributos essenciais da Atenção Primária e a Estratégia Saúde da Família. Rev Bras Enferm. 2013; 66(Esp.):158-64.

4. Sumar N, Fausto MCR. Atenção Primária à Saúde: a construção de um conceito ampliado. J Manag Prim Health Care. 2014; 5(2):202-12. 
5. Silva LA, Casotti CA, Chaves SCL. A produção científica brasileira sobre a Estratégia Saúde da Família e a mudança no modelo de atenção. Cienc Saude Colet. 2013; 18(1):221-32.

6. Gervas J, Fernandez-Perez M. Uma Atenção Primária forte no Brasil. Relatório sobre como fortalecer os acertos e corrigir as fragilidades da Estratégia de Saúde da Família [Internet]. [acesso em 2017 fev 25], [aprox. 99 páginas]. Disponível em: http://www.sbmfc.org.br/media/file/documentos/relatoriofinal_portugues.pdf

7. Barreto JOM, Nery IS, Costa MSC. Estratégia Saúde da Família e internações hospitalares em menores de 5 anos no Piauí, Brasil. Cad Saúde Pública. 2012; 28(3):515-26.

8. Leão CDA, Caldeira AP. Avaliação da associação entre qualificação de médicos e enfermeiros em atenção primária em saúde e qualidade da atenção. Cienc Saude Colet. 2011; 16(11):4415-23.

9. Ministério da Saúde do Brasil. Manual do instrumento de avaliação da atenção primária à saúde: primary care assessment tool pcatool - Brasil. Brasília: Ministério da Saúde; 2010.

10. Segall-Corrêa AM, Pérez-Escamilla R, Maranha LK, Sampaio MFA, Yuyama L, Alencar F, et al. Acompanhamento e avaliação da segurança alimentar de famílias brasileiras: validação de metodologia e de instrumento de coleta de informação. Campinas: Universidade Estadual de Campinas/Organização Pan-Americana da Saúde/Ministério de Saúde; 2003. (Relatório Técnico).

11. Harzheim E, Starfield B, Rajmil L, Dardet CA, Stein AT. Consistência interna e confiabilidade da versão em português do Instrumento de Avaliação da Atenção Primária (PCATool - Brasil) para serviços de saúde infantil. Cad Saúde Pública. 2006; 22(8):1649-59.

12. Leão CDA, Caldeira AP, Oliveira MMC. Atributos da atenção primária na assistência à saúde da criança: avaliação dos cuidadores. Rev Bras Saúde Matern Infant. 2011; 11(3):323-34.

13. Malta DC, Santos MAS, Stopa SR, Vieira JEB, Melo EA, Reis AAC. A Cobertura da Estratégia de Saúde da Família (ESF) no Brasil, segundo a Pesquisa Nacional de Saúde, 2013. Cienc Saude Colet. 2016; 21(2):327-38.

14. Oliveira VBCA, Veríssimo MLÓR. Assistência à saúde da criança segundo suas famílias: comparação entre modelos de Atenção Primária. Rev Esc Enferm USP. 2015; 49(1):30-6.

15. Mesquita Filho M, Luz BSR, Araújo CS. A Atenção Primária à Saúde e seus atributos: a situação das crianças menores de dois anos segundo suas cuidadoras. Cienc Saude Colet. 2014; 19(7):2033-46.

16. Marques AS, Freitas DA, Leão CDA, Oliveira SKM, Pereira MM, Caldeira AP. Atenção Primária e saúde materno-infantil: a percepção de cuidadores em uma comunidade rural quilombola. Cienc Saude Colet. 2014; 19(2):365-71.

17. Araújo RL, Mendonça AVM, Sousa MF. Percepção dos usuários e profissionais de saúde no Distrito Federal: os atributos da atenção primária. Saúde Debate. 2015; 39(105):387-99.

18. Damasceno SS, Nóbrega VM, Coutinho SED, Reichert APS, Toso BRGO, Collet N. Saúde da criança no Brasil: orientação da rede básica à Atenção Primária à Saúde. Cienc Saude Colet. 2016; 21(9):2961-73.

19. Sala A, Luppi CG, Simões O, Marsiglia RG. Integralidade e Atenção Primária à Saúde: avaliação na perspectiva dos usuários de unidades de saúde do município de São Paulo. Saúde Soc. 2011; 20(4):948-60.

20. Silva SA, Baitelo TC, Fracolli LA. Avaliação da Atenção Primária à Saúde: a visão de usuários e profissionais sobre a Estratégia de Saúde da Família. Rev Latino-Am Enfermagem. 2015; 23(5):979-87.

21. Szwarcwald CL, Damacena GN, Souza Júnior PRB, Almeida WS, Malta DC. Percepção da população brasileira sobre a assistência prestada pelo médico. Brasil, 2013. Cienc Saude Colet. 2016; 21(2):339-49.

22. Braz JC, Mello DF, David GYM, Teixeira AS, Prado AS, Furtado MCC. A longitudinalidade e a integralidade no cuidado às crianças menores de um ano: avaliação de cuidadores. Medicina (Ribeirão Preto). 2013; 46(4):416-23.

23. Cecchetti VEM, Pedrosa MRKB, Oliveira TBRG, Silva RAP, Neusa C. Longitudinality in childcare provided through Family Health Strategy. Rev Gaúcha Enferm. 2015; 36(4):49-54.

24. Sampaio LFR. The Brazilian health system: highlighting the primary health care reform. IJPH. 2010; 7(2):359-68.

25. Nedel FB, Facchini LA, Martín M, Navarro A. Características da atenção básica associadas ao risco de internar por condiçôes sensíveis à atenção primária: revisão sistemática da literatura. Epidemiol Serv Saude. 2010; $19(1): 61-75$. 
26. Pereira FJR, Silva CC, Lima Neto EA. Condições Sensíveis à Atenção Primária: uma revisão descritiva dos resultados da produção acadêmica brasileira. Saude Debate. 2014; 38(especial):331-42.

27. Medeiros DSG, Soares DS, Duarte CSE, Oliveira TBRG, Neusa C. Avaliação do atributo integralidade na atenção à saúde da criança. Rev Gaúcha Enferm. 2016; 37(4):e57067.

28. Costa GD, Cotta RMM, Reis JR, Ferreira MLSM, Reis RS, Franceschini SCC. Avaliação da atenção à saúde da criança no contexto da Saúde da Família no município de Teixeiras, Minas Gerais (MG, Brasil). Cienc Saude Colet. 2011; 16(7):3229-40.

29. Silva JM, Caldeira AP. Modelo assistencial e indicadores de qualidade da assistência: percepção dos profissionais da atenção primária à saúde. Cad Saúde Publica. 2010; 26(6):1187-93.

\section{Notas}

* $\quad$ Artigo científico de pesquisa.

\section{Licencia Creative Commons CC BY 4.0}

Como citar este artículo: Pedraza DF, Santos IS. Percepção materna do cuidado à saúde da criança na estratégia saúde da família de dois municípios da Paraíba, Brasil. Rev Gerenc Polít Salud. 2017; 17 (34): 1-15. https://doi.org/10.11144/Javeriana.rgps17-34.pmcs 